INPLASY

PROTOCOL

To cite: Lu et al.

Electroacupuncture for treating herniated lumbar disc-related sciatica: protocol for Bayesian network meta-analysis. Inplasy protocol 2020110017. doi: 10.37766/inplasy2020.11.0017

Received: 05 November 2020

Published: 05 November 2020

Corresponding author: Jun Xiong

xiongjun196071@163.com

Author Affiliation:

Jiangxi University of

Traditional Chinese Medicine

Support: No funding.

Review Stage at time of this submission: The review has not yet started.

Conflicts of interest:

The authors declare no conflicts of interest.

\section{Electroacupuncture for treating herniated lumbar disc-related sciatica: protocol for Bayesian network meta-analysis}

Lu, L1; Xiong, J2; Chen, J3.

Review question / Objective: To evaluate the effectiveness and safety of electroacupuncture treatment for herniated lumbar disc-related sciatica.

Condition being studied: Sciatica is characterized by sciatic nerve pain (pain that radiates from the low back to below the knee), paresthesia (mostly numbness and tingling), and muscle weakness in the affected leg or foot.

Information sources: Electronic databases, contact with authors, trial registers, or grey literature. Pubmed, Embase, Cochrane Library, Chinese Biomedical Literatures Database(CBM), China National Knowledge Infrastructure (CNKI) , WangFang Database (WF), Chinese.

INPLASY registration number: This protocol was registered with the International Platform of Registered Systematic Review and Meta-Analysis Protocols (INPLASY) on 05 November 2020 and was last updated on 05 November 2020 (registration number INPLASY2020110017).

\section{INTRODUCTION}

Review question / Objective: To evaluate the effectiveness and safety of electroacupuncture treatment for herniated lumbar disc-related sciatica.

Condition being studied: Sciatica is characterized by sciatic nerve pain (pain that radiates from the low back to below the knee), paresthesia (mostly numbness and tingling), and muscle weakness in the affected leg or foot.

\section{METHODS}

Participant or population: Patients with herniated lumbar disc-related sciatica. 
Intervention: Electroacupuncture, or electroacupuncture with other routine treatments(western medicine, exercise and so on).

Comparator: Western medicine, placebo, sham electroacupuncture, exercise, no treatment. Studies that compared acupuncture plus another therapy with the same another therapy alone will be tolerated.

Study designs to be included: We will include Randomized controlled trials (RCTs) involved patients with herniated lumbar disc-related sciatica irrespective of gender, race, age, and setting.

Eligibility criteria: Subjects: patients with herniated lumbar disc-related sciatica, age and sex were not restricted. Intervention measures: Electroacupuncture ,or electroacupuncture with other routine treatments (western medicine, exercise and so on)were used as the intervention measures in the treatment group, while sham electroacupuncture or other non-sham electroacupuncture therapy was used as the intervention measures in the control group Outcome measures: the visual analogue scale(VAS), Abbreviated Acceptability Rating Profile (AAPR).

Information sources: Electronic databases, contact with authors, trial registers, or grey literature. Pubmed, Embase, Cochrane Library, Chinese Biomedical Literatures Database(CBM), China National Knowledge Infrastructure (CNKI), WangFang Database (WF), Chinese.

Main outcome(s): the visual analogue scale(VAS), Abbreviated Acceptability Rating Profile (AAPR).

Quality assessment / Risk of bias analysis: Included randomised studies will be assessed for risk of bias by two independent raters(Lunbin Lu and Jun Chen) using the Cochrane Collaboration's tool for assessing risk of bias in randomised trials. Any disagreements will be resolved through discussion or consultation with a third reviewer(Jun Xiong).

Strategy of data synthesis: Data synthesis will be conducted with RevMan V.5.4 software provided by the Cochrane Collaboration. Before data meta-analysis, we measure the heterogeneity with a standard test. Depending on the level of heterogeneity, those studies with high heterogeneity $(p>0.10)$ will use fixed-effect model. We will use the RR for dichotomous data and SMD for continuous data and mean difference with $95 \%$ Cls. Those studies with low heterogeneity $(p=0.10)$, we use the random-effect model. Subgroup or sensitivity analysis will be performed if necessary. We will use qualitative analysis if there is excessive data heterogeneity.

Subgroup analysis: If the necessary data are available, subgroup analysis will be carried out according to different factors as follows: 1. Control interventions (eg, sham electro-acupuncture, no treatment, oth er TCM treatment or non-TCM treatment). 2. Type of acupuncture and moxibustion (eg, needle acupuncture, auricular acupuncture, heat-sensitive moxibustion, thunder fire miraculous moxa roll, warm needling moxibustion, suspended moxibustion or mild moxibustion).

Sensibility analysis: To assess the influence of each individual study, leave-one-out sensitivity analysis was performed iteratively by removing one study at a time to confirm that the findings were not influenced by any single study.

Country(ies) involved: China.

Keywords: herniated lumbar disc-related sciatica; electro-acupuncture; sciatica; protocol ; Bayesian network meta-analysis.

Contributions of each author:

Author 1 - Lunbin Lu - The author drafted and improved the manuscript.

Author 2 - Jun Xiong - Revise this protocol; search strategy.

Author 3 - Jun Chen - Data collection; analysis of results. 